\title{
Sensor Networking Testbed with IEEE 1451 Compatibility and Network Performance Monitoring
}

\author{
D. Gurkan, X. Yuan, D. Benhaddou, F. Figueroa*, and J. Morris* \\ Department of Engineering Technology \\ University of Houston, \\ * NASA Stennis Space Center \\ dgurkan@uh.edu
}

\begin{abstract}
Design and implementation of a testbed for testing and verifying IEEE 1451-compatible sensor systems with network performance monitoring is of significant importance. The performance parameters measurement as well as decision support systems implementation will enhance the understanding of sensor systems with plug-and-play capabilities. The paper will present the design aspects for such a testbed environment under development at University of Houston in collaboration with NASA Stennis Space Center SSST (Smart Sensor System Testbed).

\section{INTRODUCTION}

There is an ongoing progress in the sensors and networking technologies in order to realize a network of sensors working harmoniously similar to the chips on a motherboard of a PC. In this respect, the sensors will not be able to reach the level of integration unless they have embedded networking capabilities [1]. These capabilities have been implemented in a proprietary manner in various systems by the sensor networking companies. However, there is a strong push in the industry to harmonize the standards that enable networking and data acquisition of sensors. The need comes from reaching the ultimate goal for networked smart sensors: web access that can enable a distributed decision system.

The distributed system would be possible by following the path of the networked PCs towards creating the internet: plug-and-play capabilities of PCs paved the way towards making the internet what it has become today. Similarly, sensors with plug-and-play capabilities will enable an autonomous system to be up and running virtually for every kind of sensing application. Transducer Electronic Data Sheets (TEDS) will play an equivalent role to the drivers for PC equipment in realizing this mission.

Communication protocols that integrate the TEDS information into the protocol flow control will deliver the critical differentiated services to the network in order to guarantee an upper bound on the network latency. The control networks will emerge as more dedicated networks with strict latency bounds. Request-response and publishsubscribe types of networking paradigms will exist concurrently in a sensor networking environment [2]. Information fusion that takes into account of a random network delay with an upper bound is critical in sensor networking. Although it is desired to guarantee a deterministic delay, testbeds will enable the analysis of this delay in detail towards preparation for the worst case scenario [3]. In addition to the efforts to create time-synchronized (not only by network events) through IEEE 1588 [4], there are experimental evaluations of potential sensor networks for their synchronization capabilities [5].

This paper is on the design of a testbed system to test and verify IEEE 1451 compatible sensor systems with networking performance monitoring.

\section{SSST NETWORK BACKBONE AND HIGH LEVEL FUNCATIONALITIES}

There has been a relatively long history in distributed monitoring and decision support 
system in control and systems research community [6, 7], and in wired or wireless networking in communication research community. However, the control research community usually is not concerned about network and communication parameters such as details in channel initialization, routing, reconfiguration, and power and topology constraints. Also, the control and decision support systems should be aware of errors caused by networking such as channel errors, delays, and packet losses. Recent research starts to make simple assumption such as error-free communication channels with capacity constraint [8], and research about the robustness of the decision support system to the loss of communication links [9]. The testbed presented here aims to bridge the gap between the two research communities.

When designing and implementing such as testbed for plug-and-play intelligent sensors, we make use of a computer networking testbed as a stand-alone networking backbone with various architectural capabilities. The tesbed networking backbone architecture is presented in Figure 1.

The infrastructure backbone has state-of-the-art networking gear for wireless access and virtual networking with fiber-optic support. Initially, offthe-shelf IEEE 1451 compatible smart sensors will be connected to the backbone through wired and wireless connectors while the factory specifications are being shared by the gateway. Eventually, the testbed will emerge as an autonomous network of sensors with automated TEDS management and a central intelligence unit with integrated system health management (ISHM) functions.

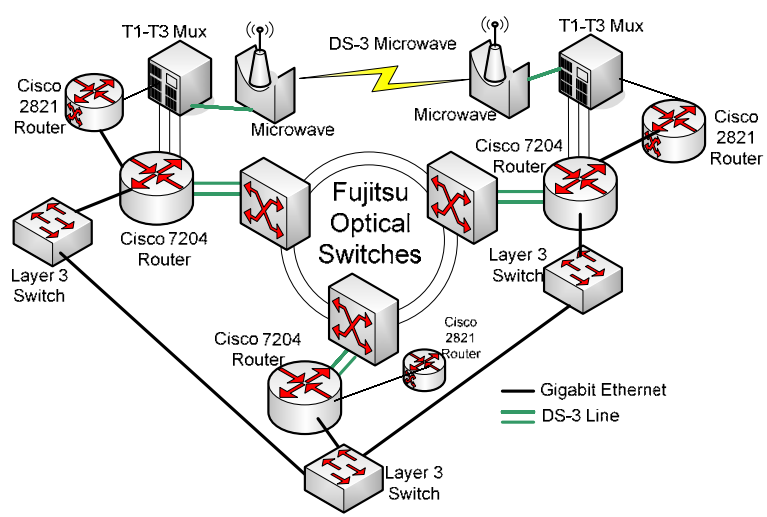

Figure. 1. Networking infrastructure backbone for the sensor testbed.

This first-of-its-kind testbed will provide up-todate data and knowledge base on the high level functionalities of a sensor network tested we identified as shown in Figure 2.

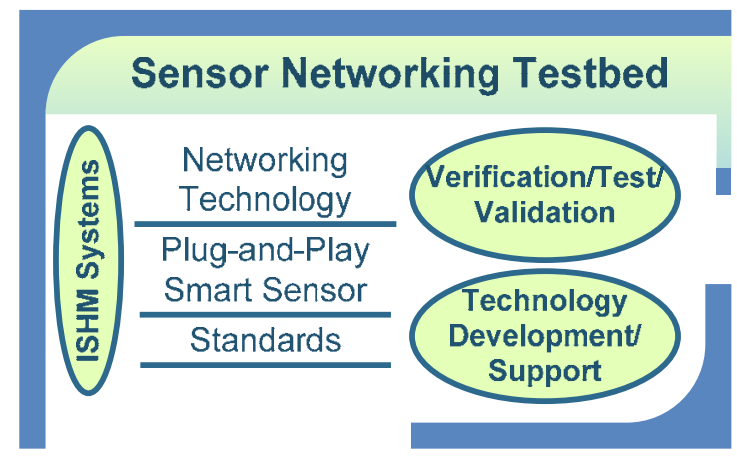

Figure 2. High level function chart of the testbed.

\section{SSST MAIN TASKS}

As presented in [10], a control loop takes into account of the network delay as shown in figure 3. The control networking performance analysis will include the protocol and standards parameters such as TEDS information delivery and updates, reconfiguration and calibration needs of smart sensors, and response timing in the network. The on-the-fly calibration and reconfiguration requirements will become as important as the data transfer with minimal and bounded latency.

Based on the high level functionalities identified, the tested is designed with NASA engineers to complement the functionalities of the ISHM system for the following tasks: 


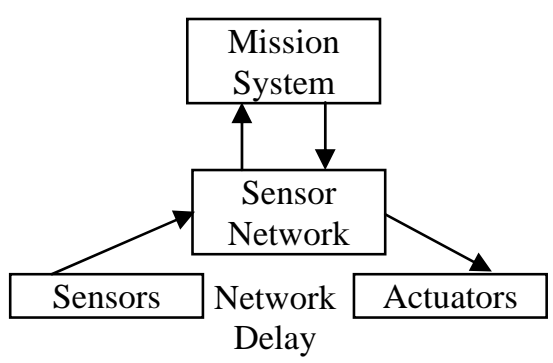

Figure 3. Control network response loop.

(i) Off-the-shelf plug-and-play sensor verification, testing, and evaluation: The standards on sensor networking have been harmonizing the access methods for the sensors to the networks. These standards and the available sensors in the marketplace will be cross-matched and verified using the testbed. The process will help industry in validating the requirements lists for developing smart IEEE 1451 compatible sensors.

(ii) Implementation metrics and requirements for monitoring and evaluation of capabilities in a Systems of Systems (SoS): The performance of the network will be monitored using the real-time control parameters. The testbed will provide networking performance monitoring and evaluation capability based on following indices: network latency, throughput, synchronization, and deterministic and random behaviors.

(iii) Assessment of the flexibility and integrability of different sensor systems. The standards of sensor interfacing will be tested and verified for SoS deployment over this testbed. Assessment methods and test scenarios will be developed in order to have a uniform performance evaluation and specification validation of standardized issues in sensor networks.

\section{SSST DEVELOPMENT CHALLENGES}

The testbed will provide a mechanism to assess networking architectures, smart sensor capabilities, and standardization procedures. It consists of a high-end internet backbone and offthe-shelf IEEE 1451 compatible smart components. We identified following challenges for developing and implementing such a testbed:
(1) Networking and Web Access:

a. Research and experimentation of possible candidate architectures to determine the challenges of plug-and-play sensors operating in an intelligently controlled computer network.

b. Experimentation with deterministic and best effort protocols (e.g. TCP, UDP and RTP) and study their effect on ancillary and real-time application scheduling.

(2) Smart Sensor Interfacing:

a. Health management computer system and sensor interaction (sensor communication and development standards,

b. TEDS definitions for sensors in the network.

(3) Standardization Procedures:

a. Possible networking architectures with web access will adhere to the emerging IEEE 1451 sensor interfacing standards.

b. TEDS distribution and sensor data readings will follow the IEEE 1451 standards.

\section{SSST COMPONENTS}

Besides the high-end internet backbone, the testbed being developed consists of wired and wireless networking and communication devices, and various off-the-shelf IEEE 1451 compatible smart sensor components. By supporting IEEE 1451 smart sensor standard, SSST will provide an open framework to simulate and model behaviors of distributed monitoring and control systems based on wired and wireless smart sensor network with different networking parameters configurations and various networking error scenarios.

The first version of the testbed has components for the following categories.

1. State-of-the-art wireless sensor system development kits;

2. Wireless access point with virtual private network assignments to various sensor clusters;

3. Sensors with plug-and-play capabilities; 
4. Main gateways to request information from sensors as well as to assign tasks to actuators;

5. Remote data acquisition interfacing to all of the equipment for remote monitoring and www access/hosting;

6. Sensor TEDS development software (e.g., Esensors, Inc [11]); and

7. Interface with intelligent control and decision support system such as G2 by Gensym, Inc. [12].

\section{CONCLUSION AND FUTURE WORK}

Networked sensor systems with standard plugand-play capabilities are attracting a lot of attention in the research and industry community. Specifically, IEEE 1451 standard is perceived to introduce smart sensors with plug-and-play capabilities. There is a need to develop a testbed to evaluate different architectures and protocols in IEEE-1451 enabled sensors. This testbed will play a big role in meeting this need and help quantize different performance metrics. Future work will include implementation, data collection and analysis of heterogeneous technologies in the testbed and methods to evaluate the performance of smart sensor systems.

\section{ACKNOWLEDGMENT}

The authors would like to thank NASA for funding this work under CAN agreement no. NNS06 ZBA001C.

\section{REFERENCES}

[1] T. Abdelzaher, J. Stankovic, S. Son, B. Blum, T. He, A. Wood, Chenyang Lu, "A Communication Architecture and Programming Abstractions for Real-Time Embedded Sensor Networks," IEEE Proc. of the $23^{\text {rd }}$ International Conference on Distributed Computing Systems Workshops, 2003.

[2] R. D. Schneeman, "Implementing a standardsbased distributed measurement and control application on the internet," US Dept. of Commerce, NIST Sensor Integration Group, Technical Report, June 1999.

[3] R. Hammett, M. Ferry, "A testbed for the development, demonstration and testing of information fusion systems," IEEE Proc. of the $7^{\text {th }}$ International Conference on Information Fusion, 2005.

[4] A. Moldovansky, “Application of IEEE 1588 in industrial automation and motion control systems," Rockwell Automation, Presentation in the IEEE 1588 Workshop Tutorial, October 10, 2005.

[5] W. S. Conner, J. Chhabra, M. Yarvis, and L. Krishnamurthy, "Experimental evaluation of topology control and synchronization for inbuilding sensor network applications,” Journal of Mobile Networks and Applications, vol. 10, pp. 545-562, 2005.

[6] S.C.A.Thomopoulos, R. Viswanathan, and D.K.Bougoulias, "Optimal distributed decision fusion,” IEEE Trans. Aerospace Elect. Syst., vol. 25, pp. 761-765, Sept. 1989.

[7] J.N.Tsistsiklis, "Decentralized detection," in Advances in Statistical Processing, Signal Detection, vol. 2, H.V. Poor and J.B. Thomas, Eds. Greenwich, CT:JAI, 1993.

[8] S.C.A. Thomopoulos and L.Zhang, "Distributed decision fusion with network delays and channel errors," Inform. Sci., vol. 66, no. 1, pp. 91-118, Dec., 1992.

[9] Z.B.Tang, K.R.Pattipati, and D.L.Kleinman, "Optimization of distributed detection networks: Part II generalized tree structures,” IEEE Trans. Syst., Man and Cybern., vol. 25, pp.21-42, Jan. 1995.

[10] Hong Seong Park, Yong Ho Kim, DongSung Kim, and Wook Hyun Kwon, "A scheduling method for network-based control systems,” IEEE Transactions On Control Systems Technology, vol. 10, no. 3, May 2002.

[11] Online: www.eesensors.com

[12] Online: www.gensym.com 